\title{
Methods of Parametric Optimization of Thin-Walled Structures and Parameters which Influence on it
}

\author{
Anton Kibkalo ${ }^{1, a}$, Maria Lebedeva ${ }^{2}$ and Mikhail Volkov ${ }^{1}$ \\ ${ }^{1}$ Peter the Great St. Petersburg Polytechnical University, Polytechnicheskaya st. 29, 195251, St. Petersburg, Russia \\ ${ }^{2}$ St. Petersburg State University of Architecture and Civil Engineering, 2-nd Krasnoarmeiskaya St. 4, 190005, St. \\ Petersburg, Russia
}

\begin{abstract}
The question of efficiency of thin-walled structures contains a number of contradictions. You need to select the best from all the existing structures on the criteria of optimization options. The search is conducted by varying of the parameters at parametric optimization. As a rule the aim of building structure optimization is reducing of material consumption, the labor input and cost. The costs of a particular variant of construction most full describes the given cost. There are two types of optimization parameters - immutable and varying. The result of the optimization of thin-walled beams will be a combination of parameters for each design situation in which provides the required strength and the minimum of the objective function - factory cost of production.
\end{abstract}

\section{Introduction}

The question of efficiency of thin-walled structures contains a number of contradictions. For example there is a saving of steel at a flexible wall of beam, but in the same time the high of the beam is increased. It leads to reduction of useful workshop volume or to the increasing amount of the wall panels. The erection of corrugated wall more cumbersome: there are additional process steps, increasing the amount of weld metal needed additional equipment.

Search of the best of all possible options, in terms of a given optimality criterion is called optimization. Depending on the nature of the differences of one embodiment (alternatives) from the other there are two types of optimization $[1,2]$.

At parametric optimization search is conducted by varying the process parameters that affect its state. This is the geometric dimensions and physical properties of the materials of construction components. Often when a parametric search of the best option there is a situation, when all options of combination of parameters have been exhausted, and the optimal solution is not found. In such cases we speak of improper structure of the object. Thus, structural optimization objective is to achieve the object of optimality due to the choice of its structure. In the case of building structure it is its constructive solutions (type of elements, their number, the principle of mutual location, connection type).

\footnotetext{
${ }^{a}$ Corresponding author : chelsi-1993@mail.ru
} 


\section{Methods and materials}

To turn on the contradictory nature of the optimization problem in mathematical analysis handy tool, it requires a clear statement of the problem, which traditionally consists of three components:

1. The objective function determines the optimal solution, while there are three kinds of purpose of the objective function: maximizing, minimizing or permanent limit (assignment setpoint).

2. Restrictions set immutable relationship between the permanent parameters and variable parameters, and of these dependencies following limits of varied parameters.

3. The boundary conditions indicate the extent to which may be the value of optimization parameters.

The formulated optimization problem will have a number of possible solutions that satisfy all constraints and boundary conditions. Type of objective function sets a certain number of optimal solutions.

The optimization problem arises in situations where there is no possibility to make a decision without a quantitative assessment of options (alternatives). For this assessment in the first place need to identify the purpose of optimization.

As a rule the aim of building structure optimization is reducing of material consumption, the labor input and cost. There is may be another target in depending on the specific conditions. For example, in the case of the construction of a long distance from the factory it could be reduce the cost of transportation design to on-site storage.

To formulate an optimality criterion - means to identify the signs, which should be carried out a comparative evaluation of alternatives and select the best among them. From the criterion depends on the form of the objective function, and hence the method for solving the optimization problem. Also optimality criterion affects the objectivity of the results, depending on the completeness of test parameters in the objective function.

The most accurate optimization results can be obtained by considering the problem of multicriteria, which could be taken into account several parameters. However, the transition to this task seriously complicates the solution.

To avoid such complications, complex criteria can be selected. It would cover most of the indicators characterizing the optimization goal. That criterion is the price, the basic terms of which are always the mass and the complexity of the structure of production.

The costs of a particular variant of construction most full describes the given cost [2], which include the cost of "in erection" and operating costs. In turn, the cost of "in erection" consists of the production cost (the cost of materials, implementation of manufacturing operations for the production, the costs in the factory and costs out of production) and technological installation cost (the sum of the costs of implementing operations on installation design in the construction of the building).

It is important that the optimality criterion is formed of such costs, which are amenable to fairly precise definition at the stage variant design. The complexity of the procedure of calculating the cost should be justified by the reliability of the results.

Costs of transportation and installation of construction, especially in the operation, it is difficult to define precisely because of their dependence on a number of factors not related to the merits of the problem (distance from the construction site of the manufacturer, methods of transportation, conditions and methods of installation, power construction company, the projected production process, etc.)

From this perspective, the objective results can be achieved by taking the manufacturing cost structure of production as optimality criterion.

Results of parametric optimization allow us to describe the nature of the influence of each parameter on the objective function.

Methods for calculating the technical and economic indicators, including production cost, are divided into two main groups $[3,4,5]$ :

- accrued methods based on a detailed calculation of the required rental at list prices, the calculation of operational labor input; 
- analytical (enlarged) methods with the use of formulas, tables and nomograms, approximating statistics.

In the present study to cover different design situations is preferred analytical method that allows more compact to formulate an objective function. This approach corresponds to the method proposed by JM Likhtarnikov [6] in the 70-ies of XX century. Factory structure manufacturing costs are determined according to the complexity of the design weight of PI by the formula:

$$
C_{u}=\left[G_{k} \cdot C_{\text {ом }}+T \cdot a \cdot\left(1+K_{\mathrm{нp}}\right)+G_{k} \cdot H\right] \cdot K_{u} \cdot K_{\mathrm{p}}
$$

where $G_{k}-$ structural mass;

$\mathrm{C}_{\mathrm{OM}}$ - cost of materials;

$\mathrm{T}$ - the total complexity of design;

a - hourly wages of workers;

$\mathrm{K}_{\mathrm{нр}}$ - coefficient taking into account of overheads;

$\mathrm{H}$ - conditionally fixed costs for maintenance and production management;

$\mathrm{K}_{\mathrm{u}}, \mathrm{K}_{\mathrm{p}}$ - coefficients taking into account the profitability of the construction and deductions for NCI, bonuses, etc.

Object parameter optimization called all the characteristics of an object, describing his condition. The total number of parameters can be divided into two groups: the variable parameters that change with a certain pitch in a certain range; unchangeable parameters that describe the source data for the design. The true solution of the problem of optimization is such a solution, in which the combination of the optimal parameters does not conflict with the embodiment of the project in practice. To do this, formulated the boundary conditions (border regions of varying parameters), set iteration step, which provides product mix parameters.

\section{Optimization parameters}

\subsection{Immutable parameters}

\subsubsection{Span}

Beams with a flexible or corrugated wall coverings used as a truss for buildings or roof purlins. Span length, which should be overlapped by beam depends on the intended manufacturing process. But in any case the span length should be a multiple of the module of $3 \mathrm{~m}$ or $6 \mathrm{~m} \mathrm{[7].} \mathrm{Length} \mathrm{of} \mathrm{the} \mathrm{beam} \mathrm{is}$ determined by step of bearing trusses and beams of coverage (transverse frames), which usually equals the modular size of wall panels, covering panels, that $6 \mathrm{~m}$ or $12 \mathrm{~m}$. If the pitch of bearing frames exceeds the specified values for overlapping used secondary structures usually through the section. From the variety of buildings is emited large-span buildings with 36-120 meters span length: industrial buildings for manufacturing or assembly of large products - aircraft, ships and other equipment, the storage of various equipment: aircraft hangars, depots for electric, garages, etc. Already during the flight $40 \ldots 60 \mathrm{~m}$ in the middle of the span large internal forces develop and the use of continuous wall structures is inefficient compared with through structures (farms).

\subsubsection{Load}

To limit an extensive range of possible values of the load, consider some design situations. The use of thin-walled beams is most likely in the projects with the use of modern lightweight materials. Usually galvanized corrugated decking steel plays the role of a light carrier flooring, which is laid on the girders, made of galvanized cold-formed channel section profiles. The use of non-combustible materials contributes to reduction of mass insulation; these materials has small volume weight and the minimum value of thermal conductivity, such as extruded polystyrene foam. The need for insulation, 
type and thickness depends on the process that determines the required indoor temperature and climatic region. From climatic region also depends on the snow load. Weight of sheeting and runs depends from steps transverse frames. Boundary conditions "load" parameter defined extreme combinations of input data.

\subsection{Variable parameters}

\subsubsection{Steel grade}

As the mechanical properties of steel is conventionally divided into three groups: normal strength (C235 ... C285, Ry <Ry 40 kN / cm2), high strength (C345 S390 ... $29 \mathrm{kN} / \mathrm{cm} 2<\mathrm{Ry} 40 \mathrm{kN} / \mathrm{cm} 2)$. The last class of steels ineffective for thin-walled beams due to their low plasticity.

\subsubsection{The relative height of the beam and wall flexibility}

The smallest conditional flexibility is limited by the rules [8] $\overline{\lambda_{\mathrm{w}}} \geq 6$, which corresponds to the physical flexibility of the wall $\lambda_{\mathrm{w}}=\frac{\mathrm{h}_{\mathrm{w}}}{\mathrm{t}_{\mathrm{w}}}=250$.

Wall height $\mathrm{h}_{\mathrm{w}}$ - the width of rolled sheet, which is determined by the length of the barrel of the working rolls of sheet rolling mills. Limiting the length and width of the sheets depends on the thickness of rolled [20] (to avoid death and curvatures at the handling and storage). In the case of steel coils thinnest limited only by the width of the rolled products.

\subsubsection{The relative length of the compartment}

The minimum length of the compartment limited by the condition of using supercritical reserve bearing capacity of the thin wall. Stress which corresponds to the emergence the diagonal band in the wall (2):

$$
\tau_{c r}=10,3 \cdot\left(1+\frac{0,76}{\mu^{2}}\right) \cdot \frac{R_{S}}{\bar{\lambda}_{w}^{2}},
$$

If the length of the compartment is less than half the height of the wall $(\mu<0,5)$, the value of the critical shear stresses close to the ultimate stress. In other words, supercritical bearing capacity of the flexible wall significantly (up to three times) reduced when $\mu<0,5$.

\subsubsection{Crimping parameters}

The complexity of the search for the optimum size of the corrugations: variation of the relative dimensions of the corrugations $\left(\frac{f}{a} ; \frac{f}{t}\right)$ has a contradictory effect on the shear strength of the wall. Increase of the rigidity of the corrugation doesn't always contribute to hardening of the walls. Moreover, the size of the different shape of the corrugations has a different effect on the strength of the wall.

Optimization of parameters of corrugation is facilitated by the lack of influence of height of the wall on the critical shear stresses.

Maximum lateral force of the corrugated wall bounded on one hand of loss of stability; on the other hand - the development of plastic deformation in the wall (shear strength steel). This means that the corrugations of the walls need not be very gentle, low rigidity, but at the same time extremely rigid to the critical stress not exceed the yield strength of steel [9]. High intensity crimping results in excessive overspray, wear plate-bending equipment and welding tractors.

To make the best decisions it makes no sense to consider all the values of the objective function corresponding to each of the optimization parameters. Only those combinations of variable parameters 
are interesting for the analysis that are able to provide a building structure the required strength and stiffness without overspending material (excessive safety margin).

Of course, along with these requirements to the designed device and imposed other requirements: aesthetics, compactness, manufacturability, and so on. However, such requests are usually difficult to formalize and submit to the mathematical notation, a qualitative assessment of an expert required to meet them [10-14].

The result of the optimization of thin-walled beams will be a combination of parameters for each design situation in which provides the required strength and the minimum of the objective function factory cost of production.

\section{Conclusions}

Results of parametric optimization will describe the nature of the influence of each parameter on the objective function.

1. The use of high-strength steel leads to a rise in the cost of thin-walled beams at $18-30 \%$. Meanwhile, the mass of construction can be reduced by $30-60 \%$, that in some cases may be a very valuable asset. However, the use of higher strength steel helps reduce the cross-sectional area zones, thereby increasing the maximum deflection and leads to some overspray.

2. Increasing of relative height of beam leads to economies of steel until to a certain limit, after which the mass is increasing due to the necessity of more powerful devices belts.

3. Increasing the flexibility of the wall helps to alleviate the beam. However, the range of values for which the weight is reduced effectively, not so great.

4. Relative compartment length affects its shear strength: the weaker the compartment, the greater its length. As the reduction in the number of compartments (increasing their length) beam becomes easier and less time-consuming to manufacture.

5. Changing crimping parameters affect the labor and cost of construction. Tightening of corrugations makes sense only in cases of unstable form of exhaustion bearing capacity - at a flexible wall with gentle corrugations ultimate shear force is determined by the critical stress. In other cases, the shear strength is determined by the tensile strength of steel and material consumption to improve the stability of the corrugations is irrational.

6 . In most cases, the cost and complexity grow proportional to the mass.

\section{References}

1. I. P. Norenkov, Fundamentals of computer-aided design (2002)

2. M. R. Garifullin, N. I. Vatin, Construction of Unique Buildings and Structures, 21, 32-57 (2014)

3. V. V. Kuznetsov The steel structures of buildings and structures: a handbook designer (1998)

4. A. I. Kolesov, A. A. Lapshin, A. V. Valov, Privolzhsky Scientific Journal, 1, 28-33 (2007)

5. N. I. Vatin, V. A. Rybakov, StroyPROFIL, 56, 60-63 (2007)

6. Y. M. Likhtarnikov Variant design and optimization of steel structures (1979)

7. GOST 28984-91. Modular coordination of dimensions in construction (1991)

8. Steel construction. The updated edition of SNiP II-23-81: SP 16.13330.2011 (2011)

9. Y. I. Olkov, A. S. Poltoradnev, Proceedings of the International Congress "Science and Innovation in modern construction - 2012" International scientific-practical conference on the scientific direction "Actual problems of modern construction and ways to effectively address the" Ph. - St. Petersburg, (2012)

10. V. Z. Vlasov, Thin elastic rods (1959)

11. R. Kamnik, B. Kovačič, B. Pribicević, A. Đapo, Geodetski List, 69 (3), 171-188 (2015)

12. B. Kovačič, R. Kamnik, A. Štrukelj, N. Vatin, Procedia Engineering, 117 (1), 800-806 (2015)

13. L. Lazarević, M. Kovačević, Z. Popović, Facta Universitatis, Series: Mechanical Engineering, 13 (2), 133-141 (2015)

14. B. Kovačič, R. Kamnik, M. Premrov, Survey Review, 43 (320), 150-161 (2011) 